\title{
Voltage dependent mineral deposition mediated by hydrothermal vent microbes
}

BRANDON C ENALLS ${ }^{1}$, JENNIFER DELANEY ${ }^{1}$ AND PETER GIRGIUS ${ }^{2}$

\author{
${ }^{1}$ Harvard Univeristy \\ ${ }^{2}$ Harvard University
}

Presenting Author: benalls@g.harvard.edu

Deep sea hydrothermal vent chimneys have reduction potentials much lower than the ambient seawater, due to the oxidation of vent derived reductants at the interface between the hydrothermal fluid and the interior chimney walls and the subsequent transfer of electrons through semi-conductive metal sulfide matrices that comprise the chimneys. It is known that vent mineralogy can affect the microbial community composition in vent chimneys, but it is less clear how microbes affect the distribution of minerals in these environments. Previous work using bioelectrochemical systems and X-ray fluorescence has shown that electrodes poised at anodic potentials exhibited greater metal sulfide deposition in systems containing live vent inoculum versus killed controls. We expand upon this work by conducting incubations at two more cathodic electrical potentials, representing other electrochemical states and microbial niches within vent chimneys. We found that electrodes poised at $+39 \mathrm{mV}$ vs. SHE had more deposition of minerals containing iron and copper than zinc minerals in systems containing living and killed microbes. Control electrodes that were unpoised had more deposition of zinc minerals than minerals containing iron and copper. When poised at $-200 \mathrm{mV}$, electrodes in systems with living microbes had less overall deposition than control treatments. These data, taken together with previous work, suggest that microbes may influence the deposition of metal sulfides in a voltage dependent manner. This deposition data, combined with chronoamperometric data and 16S microbial community analyses, have allowed us to characterize the electrochemical interactions between microbes, minerals, and conductive substrates in these environments over a range of electrical potentials. 